\title{
Huiles et corps gras végétaux : ressources fonctionnelles et intérêt nutritionnel
}

\author{
Odile MORIN $^{1}$ \\ Xavier PAGÈS-XATART-PARÈS ${ }^{2}$ \\ 1 ITERG - Institut des Corps Gras, \\ 11 rue Gaspard Monge, \\ 33600 Pessac, \\ France \\ $<$ O.MORIN@iterg.com> \\ ${ }^{2}$ ITERG - Institut des Corps Gras, \\ Parc Industriel Bersol, \\ 33600 Pessac, \\ France
}

\begin{abstract}
Vegetable oils \& fats: functional resources and nutritional interest The wide variety of oleaginous resources potentially provides to industrial users a wide range of fats and oils, and so, a large range of properties. But less than 10 vegetable fats and oils - such as palm and its fractions, soybean, rapeseed, sunflower, cotton, peanut and olive oils - represent more than $90 \%$ of the world production. So, these few vegetable fats and oils will have to respond simultaneously to numerous expectations, depending on the user, such as providing optimized nutrients and offering the texture looked forward or resisting to an established cooking temperature. Innovation (use of new variety with modified compositions, existing "niche" oil or completely new resource) could be an option to solve a specific issue, even if economical or regulatory constraints may not favor such alternatives. After summarizing vegetable fats and oils composition and the properties of fatty acids, this paper presents not only optimized processes allowing extraction of compounds of interest, but also processes for the conversion and enlargement of oils and fats functionalities in order to answer to the different and specific expectations of industrial users and consumers.
\end{abstract}

Key words: vegetable fats and oils, composition, fatty acids, tocopherols, micronutrients, functionality

La grande diversité des ressources oléagineuses met potentiellement à disposition des différents secteurs utilisateurs une grande variété de corps gras. Cependant, $90 \%$ des productions mondiales se concentrent sur une petite dizaine d'huiles (palme et dérivés, soja, colza, tournesol, coton, arachide, olive) que les secteurs consommateurs vont utiliser selon les fonctionnalités qu'ils recherchent. Celles-ci sont variées et plusieurs peuvent être requises simultanément: apport optimisé de nutriments et texturation ou résistance à la température, par exemple. Par ailleurs, l'évolution des connaissances sur l'impact des régimes alimentaires ou de certains composants (à partir des résultats d'études épidémiologiques ou d'intervention, de recherche sur les métabolismes) et les recommandations nutritionnelles qui en découlent à terme, incitent à faire évoluer certaines habitudes alimentaires. Ces orienta- tions favorisent l'innovation impliquant selon les cas, soit la mise au point de procédés nouveaux (fonctionnalité modifiée), soit l'emploi d'une autre ressource (nouvelles variétés de compositions différentes, recherche d'une alternative référencée et disponible ou innovante issue du " sourcing ", avec les freins bien connus que peuvent générer par exemple, la création d'une filière d'approvisionnement ou les procédures réglementaires liées à l'introduction de tout nouvel aliment sur le marché). Enfin, de grandes productions mondiales peuvent, de manière conjoncturelle ou durable, souffrir d'une image dépréciée auprès de certaines régions de consommation; de telles situations sont aussi susceptibles de conduire à la recherche d'alternatives dont la complexité fait naître de nouveaux défis.

Les options répondant à ces enjeux intègrent la variété des compositions disponibles et les propriétés ou fonctionnalités qu'elles impliquent, les possibilités offertes par la technologie et les procédés d'obtention et de transformation pour mieux adapter une matière première à ses utilisations, avec les exigences requises en matière de qualité et de sécurité sanitaire.

\section{Compositions en acides gras et autres nutriments}

Un corps gras (huile ou graisse) est composé d'une grande variété de constituants que la figure 1 présente de façon panoramique ; les triglycérides sont très largement majoritaires (95-99 \%) : ils sont composés de glycérol (3-5\%) et d'acides gras (90-95\%). D'autres constituants sont naturellement présents en plus faible quantité : des lipides à caractère polaire tels que les phospholipides $(0,1-0,2 \%)$ et des composés dits

Pour citer cet article : Morin O, Pagès-Xatart-Parès X. Huiles et corps gras végétaux : ressources fonctionnelles et intérêt nutritionnel. OCL 2012 ; 19(2) : 63-75. doi : 10.1684/ocl.2012.0446 


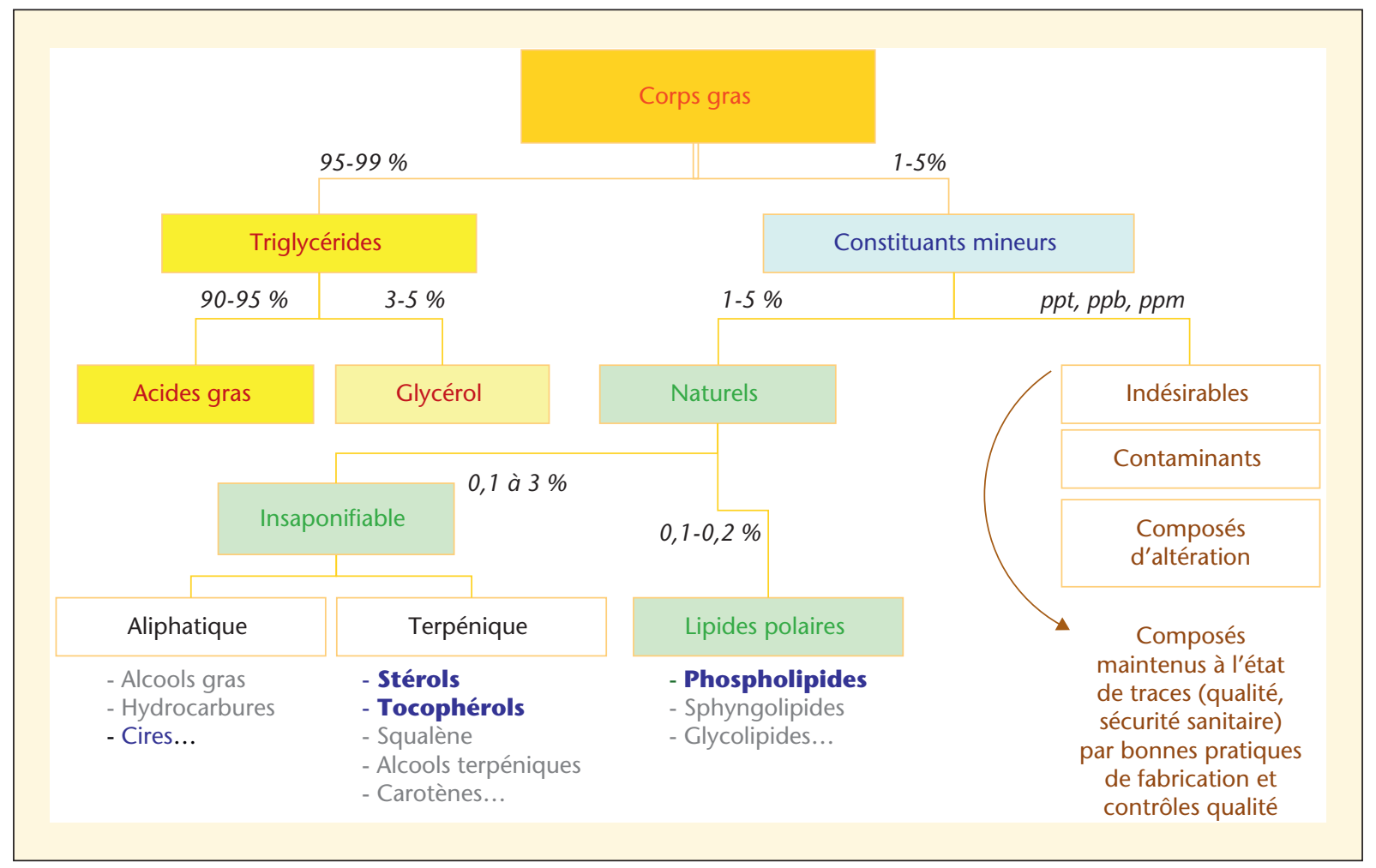

Figure 1. Composition panoramique des corps gras et importance relative des principales classes de composés.

insaponifiables appartenant à une fraction non glycéridique $(0,1$ à $3 \%)$ principalement représentés par les stérols et les tocophérols \& tocotriénols mais contenant également des caroténoïdes, des alcools terpéniques, du squalène, des composés phénoliques, etc.

\section{Compositions en acides gras}

Selon leurs compositions en acides gras, les corps gras d'origine végétale (huiles, graisses, beurres végétaux) se répartissent entre différentes familles. Selon les critères de caractérisation de ces familles, elles peuvent être plus ou moins nombreuses (Dubois et al., 2008) mais il en existe quatre principales (figures $2 A, B$, et C) :

- famille oléique où cet acide gras, principal représentant des acides gras mono-insaturés (AGMI), est majoritaire : huiles d'olive, d'arachide, de noisette, les variétés de tournesol et de colza riches en acide oléique et l'huile de colza ellemême ;

- famille linoléique où cet acide gras (C18:2 oméga-6), acide gras polyinsaturé (AGPI), est majoritaire : huiles de soja, de tournesol, de germe de maïs et de pépins de raisin ;

- famille $\alpha$-linolénique où cet acide gras (C18:3 oméga-3/AGPI) est présent en quantité significative : huiles de colza, de soja, de noix et de lin où cet acide gras est majoritaire ;

- famille des corps gras riches en acides gras saturés (AGS) avec leurs principaux représentants (C12:0, C16:0, C18:0) présents en quantité moyenne à forte : les huiles de palme, les huiles de palmiste et de coprah riches en acide laurique (C12:0), le beurre de cacao et pour comparaison la matière grasse de beurre.

Les huiles de la famille $\alpha$-linolénique autres que le lin se distinguent entre elles par leur acide gras majoritaire : I'acide oléique pour I'huile de colza et l'acide linoléique pour les huiles de soja et de noix. L'huile de sésame contient presque autant d'acide oléique que d'acide linoléique. Les huiles des familles oléique, linoléique et $\alpha$-linolénique contiennent en moyenne de 8 à $15 \%$ d'AGS, I'arachide dépassant cette fourchette avec environ $20 \%$ (ou plus). Pour mémoire, la figure $2 B$ rappelle les valeurs des apports nutritionnels conseillés (ANC, 2010) exprimées pour chaque catégorie d'acide gras en \% de l'apport lipidique total; c'est ici l'occasion de rappeler que ces recommandations doivent s'entendre au niveau d'une alimentation globale (non d'un produit isolé) et d'une population (non d'un individu).

Les tableaux 1, 2 et 3 donnent les compositions détaillées par acide gras d'une sélection un peu plus large $d$ 'huiles et de corps gras végétaux que celles des figures commentées ci-dessus.

\section{Les autres nutriments :}

tocophérols, phytostérols, caroténoïdes, composés phénoliques...

Les tocophérols et tocotriénols (ou tocols) regroupés sous le nom générique de vitamine $E$, sont deux grandes familles présentant chacune quatre formes différentes qui n'ont pas toutes la même activité biologique : pour les tocophérols la forme alpha est la plus active (100\%), les formes beta et gamma présentent des activités plus faibles (respectivement $50 \%$ et $10 \%$ ) et la forme delta ne 


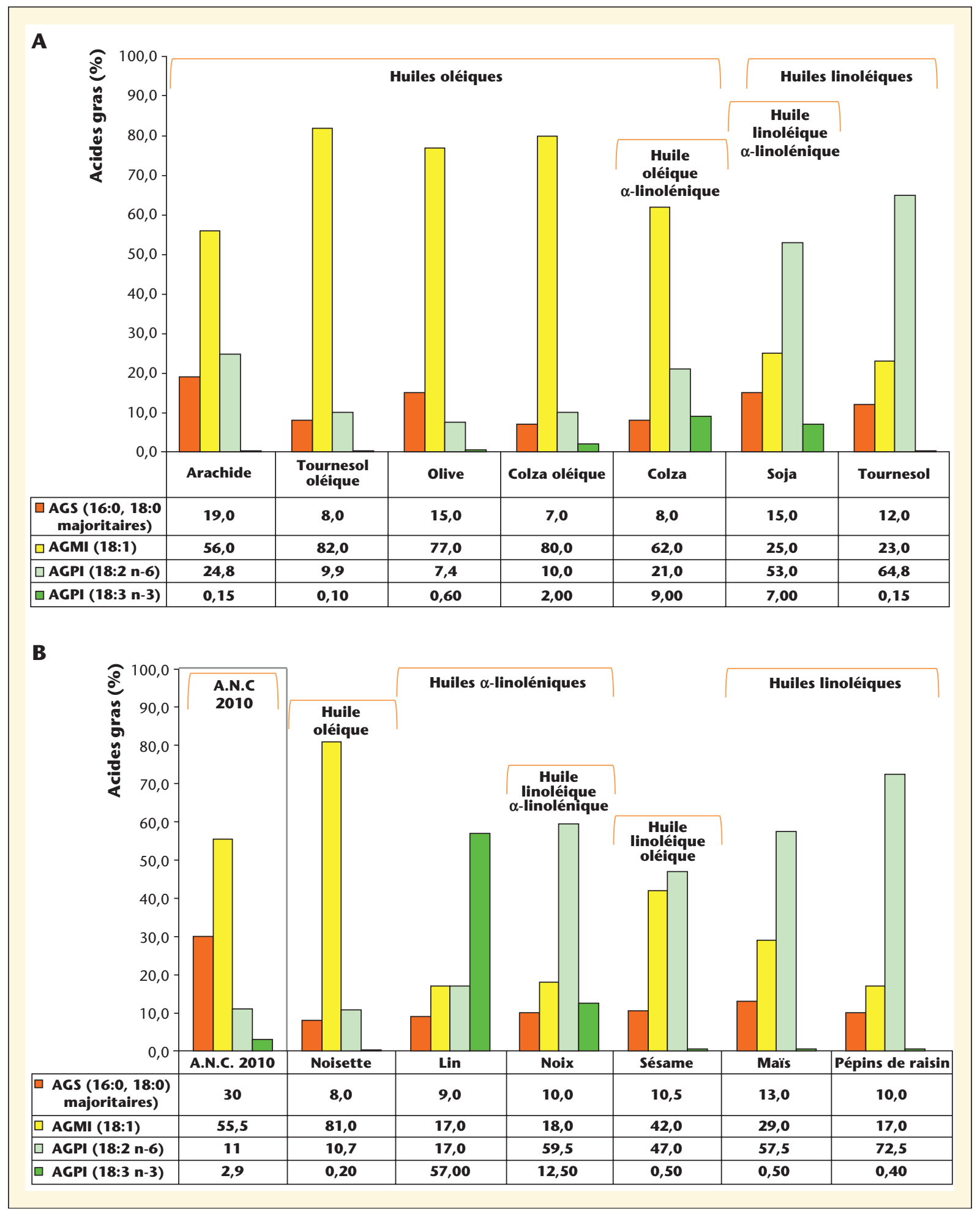

Figure 2. $A, B, C)$ Classement de quelques huiles végétales par catégorie d'acides gras (saturés, monoinsaturés, polyinsaturés linoléique et $\alpha$ linolénique) - Familles et sous-familles.

possède qu'un très faible pouvoir vitaminique $E$. Ils jouent un rôle d'antioxydant biologique, protecteur des acides gras polyinsaturés au niveau des membranes cellulaires et des lipoprotéines. Leur pouvoir antioxydant est reconnu et contribue à protéger de nombreux produits formulés (additifs
E306 à E309) et les huiles végétales qui en contiennent naturellement. Les huiles végétales en effet, mais aussi les céréales et les fruits sont les principales sources 


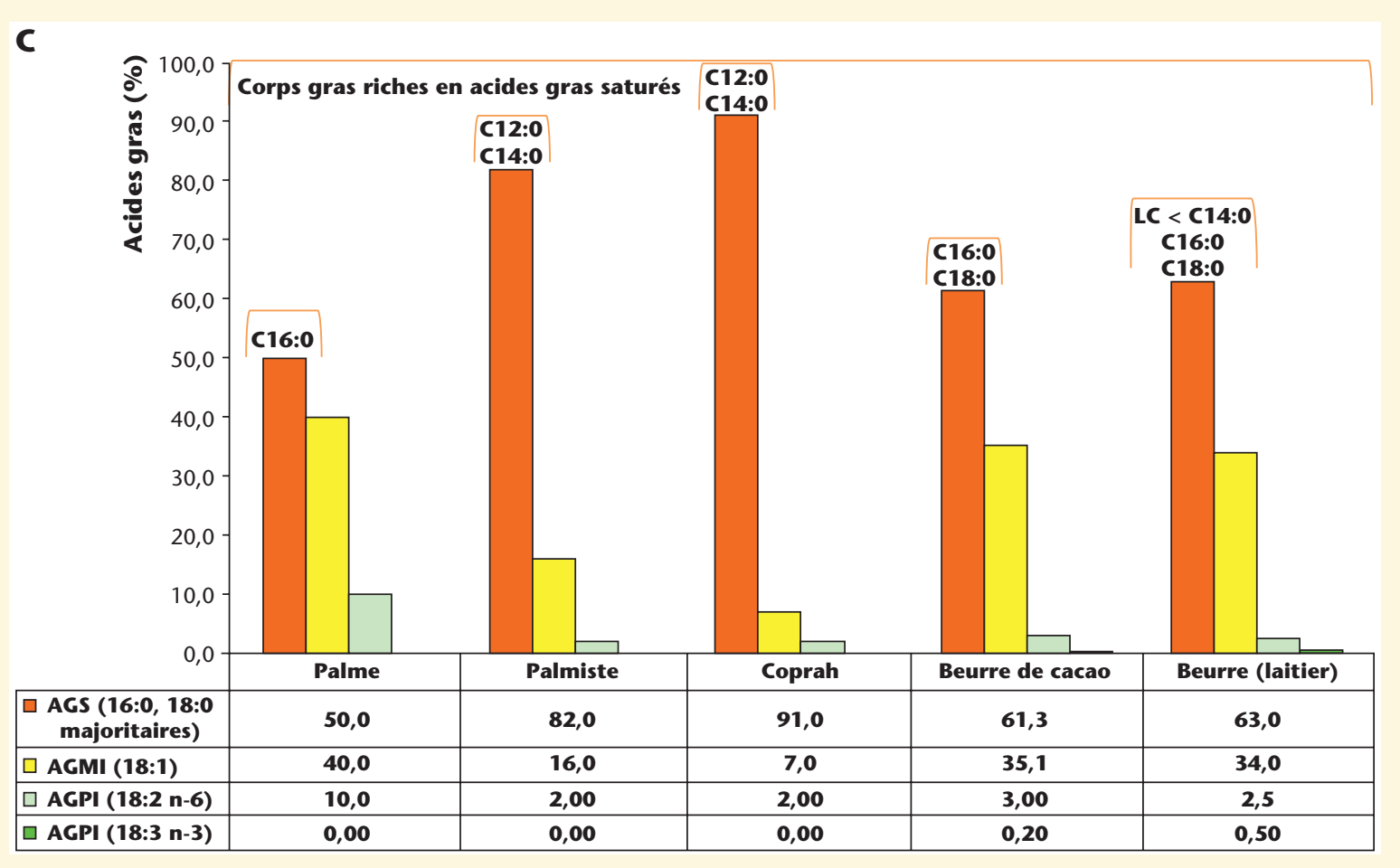

Figure 2. (Suite)

$d^{\prime}$ apports en vitamine $E$ dont les formes les plus fréquemment rencontrées sont les formes $\alpha$ - et $\gamma$-tocophérol (Landrier, 2011). Le tableau 4 rassemble les teneurs en tocophérols totaux de quelques huiles végétales, précise la forme tocophérolique majoritaire et exprime le pouvoir vitaminique $E$ en $\alpha$-tocophérol équivalent pour $100 \mathrm{~g} \mathrm{~d}$ 'huile (tenant compte de la composition en tocophérols et du pouvoir vitaminique de chaque forme) ; il apparaît que selon les formes présentes dans les huiles végétales, leur classement en pouvoir vitaminique global (rang) diffère sensiblement $d$ 'un classement par quantités croissantes de tocophérols totaux. L'apport journalier recommandé (AJR) en vitamine $E$ est de $12 \mathrm{mg}$; au sens du règlement (CE) 1924/2006 et de la directive 90/496/CE, toutes les huiles végétales peuvent $s$ 'afficher " source de vitamine $\mathrm{E}$ " puisqu'elles contiennent au moins $1,8 \mathrm{mg} / 100 \mathrm{~g}$ (15\% de l'AJR) et la plupart sont " riches en vitamine $\mathrm{E}$ " (30\% de l'AJR pour $100 \mathrm{~g}$ ).

Les phytostérols : toutes les huiles végétales en contiennent (de 0,1 à 0,5\% en moyenne) et leur structure moléculaire présente de fortes analogies avec le cholestérol. Apportés en quantité suffi- sante par I'alimentation (de 2 à $3 \mathrm{~g} / \mathrm{j}$ ), ils ont un rôle hypocholestérolémiant qui ne peut être obtenu qu'avec la consommation de produits enrichis en phytostérols (ou en phytostanols) puisque l'apport total journalier est estimé à moins de $500 \mathrm{mg}$.

Les caroténoïdes sont des pigments lipophiles sensibles à la lumière (rayonnement ultraviolet) et à la chaleur, précurseurs, tout particulièrement le trans$\beta$-carotène, de la vitamine $A$ (rétinol). Leur structure moléculaire leur confère dans certaines conditions (oxydation par photosensibilisation) un pouvoir antioxydant par désactivation de l'oxygène activé (forme singulet). Présents en forte quantité (1 à $2 \mathrm{~g} / \mathrm{kg}$ ) dans l'huile de palme rouge, les autres huiles végétales en contiennent quelques centaines de $\mathrm{mg} / \mathrm{kg}$; ces pigments sont éliminés au raffinage.

Les composés phénoliques présents notamment dans les huiles d'olive vierges (en particulier l'hydroxytyrosol, forme simple et l'oleuropéine, forme condensée) possèdent d'importantes propriétés antioxydantes. Les quantités présentes dans des huiles d'olive vierge extra de différentes provenances dépen- dent du degré de maturité des olives et sont comprises entre 100 et $400 \mathrm{mg}$ de tyrosol équivalent/kg d'huile (oleuropéine majoritaire) sans que la provenance géographique paraisse influencer les variations observées (Joffre, 2011).

Les ubiquinones ou coenzymes Q (CoQ6, $9,10)$ possèdent des propriétés d'antioxydant biologique au sein des cellules soit en inhibant la peroxydation soit en régénérant d'autres antioxydants tels que les tocophérols; ces molécules auraient ainsi un rôle protecteur vis-à-vis de la dégradation oxydative des LDLlipoprotéines, contribuant à réduire le risque cardiovasculaire (Littarru, 2011). Leur présence a été recherchée dans les aliments et après développement de méthodes d'analyse adaptées, dans les huiles végétales (Rodriguez-Acuňa, 2008), de très faibles quantités de CoQ10 (moins de $10 \mathrm{mg} / \mathrm{kg}$ ) ont été détectées dans des huiles d'olive vierges de différentes provenances (Joffre, 2011) et des teneurs très variables dans quelques échantillons d'huiles de colza, soja (non raffinées) et tournesol raffinée : de 2 à $100 \mathrm{mg} / \mathrm{kg}$ pour le CoQ9 et de 9 à 98 mg/kg pour le CoQ10 (RodriguezAcuňa, 2008). 
Tableau 1. Compositions en acides gras de quelques huiles végétales (\% des acides gras totaux).

\begin{tabular}{|c|c|c|c|c|c|c|c|c|c|}
\hline \multicolumn{2}{|l|}{ Huiles/corps gras } & \multirow{2}{*}{\begin{tabular}{|l|} 
Arachide \\
$\begin{array}{l}\text { Toutes } \\
\text { origines }\end{array}$
\end{tabular}} & \multirow[t]{2}{*}{ Cameline } & \multirow[t]{2}{*}{ Colza } & \multicolumn{2}{|c|}{ Colza oléique } & \multirow[t]{2}{*}{$\begin{array}{l}\text { Germe } \\
\text { de mais }\end{array}$} & \multirow[t]{2}{*}{ Lin } & \multirow[t]{2}{*}{ Noisette } \\
\hline Ac. gras & & & & & $\begin{array}{l}\text { Variétés } \\
\text { différentes }\end{array}$ & & & & \\
\hline \multicolumn{10}{|l|}{ saturés } \\
\hline Ac.myristique & C14:0 & - & $<0,1$ & $<0,2$ & & & $<0,1$ & - & $<0,1$ \\
\hline Ac.palmitique & C16:0 & $8-13$ & $5-8$ & $4-5$ & 3 & & $8-13$ & $4-6$ & $5-9$ \\
\hline Ac.margarique & C17:0 & $<0,1$ & $<0,1$ & - & - & & - & - & $<0,05$ \\
\hline Ac.stéarique & C18:0 & $1-4,5$ & $2-3$ & $1-2$ & 2 & & $1-4$ & $2-3$ & $1-4$ \\
\hline Ac.arachidique & C20:0 & $1-2$ & 1,5 & $<1$ & $<1$ & & $<1$ & $<0,5$ & $<0,3$ \\
\hline Ac.béhénique & C22:0 & $1,5-4,5$ & 0,5 & $<0,5$ & & & $<0,5$ & - & - \\
\hline Ac.lignocérique & C24:0 & $0,5-2,5$ & 0,2 & $<0,2$ & & & - & - & - \\
\hline \multicolumn{10}{|l|}{ monoinsaturés } \\
\hline Ac.palmitoléique & C16:1 n-7 & $<0,2$ & 0,2 & $<0,6$ & & & $<1$ & $<0,5$ & $<0,3$ \\
\hline Ac.heptadécènoïque & C17:1 & - & $<0,1$ & - & & & - & - & $<0,05$ \\
\hline Ac.oléique & C18:1 n-9 & $35-66$ & 10-24 (14-19) & $55-62(60)$ & $66-75$ & $76-80,6$ & $24-32$ & $10-22$ & $66-83$ \\
\hline $\begin{array}{l}\text { Ac.gadoléique/ } \\
\text { gondoïque }\end{array}$ & $\begin{array}{l}\text { C20:1 n-11/ } \\
n-9\end{array}$ & $0,7-1,7$ & $12-18(\underline{\mathbf{1 2}-17})$ & $1-2$ & & & $<0,5$ & $<0,6$ & - \\
\hline Ac.érucique & C22:1 n-9 & - & $2-5(\underline{1,5-4})$ & $<0,5(0,2)$ & & & - & - & - \\
\hline \multicolumn{10}{|l|}{ polyinsaturés } \\
\hline Ac.linoléique & C18:2 n-6 & $14-42$ & 12-24 (18-21) & $18-22(20)$ & $14-20$ & $11-12,6$ & $55-62$ & $12-18$ & $8-25$ \\
\hline Ac.alphalinolénique & C18:3 n-3 & $<0,3$ & $32-42(\underline{\mathbf{2 7}-35})$ & $8-10(10)$ & [3] 4,4-7,9 & $2,3-4,4$ & $<2$ & $56-71$ & $<0,6$ \\
\hline Ac.gras saturés & AGS & $12-27$ & $9-13(\underline{11})$ & $6-8$ & - & - & $10-18$ & $6-9$ & $6-13$ \\
\hline $\begin{array}{l}\text { Ac.gras } \\
\text { monoinsaturés }\end{array}$ & AGMI & $35-68$ & $24-48(\underline{39})$ & $57-65$ & 70 & 80 & $25-33$ & $11-23$ & $66-83$ \\
\hline $\begin{array}{l}\text { Ac.gras } \\
\text { polyinsaturés }\end{array}$ & AGPI & $14-42$ & $44-66(\underline{\mathbf{5 0}})$ & $26-32$ & - & - & $57-64$ & $70-80$ & $9-26$ \\
\hline & & & + C24:1 $(0,5-1)$ & & & & & & \\
\hline
\end{tabular}

\section{Familles de corps gras, structures et fonctionnalités}

Un corps gras est ainsi un ensemble complexe de constituants dont les fonctionnalités sont directement liées aux compositions et aux structures moléculaires. Les acides gras eux-mêmes se distinguent par leur longueur de chaîne (nombre d'atomes de carbone), leur nombre de doubles liaisons (aucune pour les AGS, une pour les AGMI, de deux à plus pour les $\mathrm{AGPI}$ ), la position sur la chaîne hydrocarbonée et la configuration spatiale (ou géométrie) de ces insaturations (isomères cis, trans); les principales propriétés des acides gras sont liées à leur insaturation : plus elle est élevée, plus le point de fusion diminue (augmentation de la fluidité), plus leur intérêt physiologique s'accroît tout comme leur réactivité chimique ce qui joue négativement sur la stabilité, notamment par une plus grande susceptibilité à l'oxydation. La géométrie des insaturations influence également le comportement à la fusion : à longueur de chaîne équivalente, un isomère trans $d^{\prime}$ acide gras aura ainsi un point de fusion $\left(P_{F}\right)$ intermédiaire entre ceux de son isomère cis et de l'acide gras saturé correspondant (acide stéarique, C18:0, $\mathrm{P}_{\mathrm{F}} \approx 70{ }^{\circ} \mathrm{C}$; acide oléique, C18:1 cis, $\mathrm{P}_{\mathrm{F}}=13^{\circ} \mathrm{C}$; acide élaïdique, C18:1 trans, $\mathrm{P}_{\mathrm{F}}=45^{\circ} \mathrm{C}$ ).

La structure des triglycérides qui correspond à la nature et à la position des acides gras sur le glycérol constitue de fait la composition réelle du corps gras et conditionne ses propriétés physiques (fusion, solidification) et rhéologiques ${ }^{1}$, chimiques et nutritionnelles.

La fusion, la solidification par cristallisation en des formes cristallines variées et variables (polymorphisme) selon les conditions influencent directement les propriétés rhéologiques. Le polymorphisme résulte de l'organisation spatiale des chaînes hydrocarbonées (arrangement latéral) et de l'empilement en strates des molécules de triglycérides (arrangement longitudinal); il existe deux principales catégories de variétés cristallines: celles correspondant à un

\footnotetext{
${ }^{1}$ Rhéologie : étude des phénomènes qui conditionnent l'écoulement et la déformation de la matière (plasticité, viscosité, élasticité).
} 
Tableau 2. Compositions en acides gras de quelques huiles végétales (\% des acides gras totaux).

\begin{tabular}{|c|c|c|c|c|c|c|c|c|}
\hline Huiles/corps gras & & Noix & $\begin{array}{l}\text { Olive } \\
\text { (COI/UE) }\end{array}$ & $\begin{array}{l}\text { Pepins } \\
\text { de raisin }\end{array}$ & Sesame & Soja & Tournesol & $\begin{array}{l}\text { Tournesol } \\
\text { oléique }\end{array}$ \\
\hline \multicolumn{9}{|l|}{ Ac. gras } \\
\hline \multicolumn{9}{|l|}{ saturés } \\
\hline Ac.myristique & C14:0 & - & $<0,05$ & $<0,3$ & $<0,1$ & $<0,2$ & $<0,2$ & - \\
\hline Ac.palmitique & C16:0 & $6-8$ & $7,5-20,0$ & $7-10$ & $8-11$ & $8-13$ & $5-8$ & $3,0-4,8$ \\
\hline Ac.margarique & C17:0 & $<0,1$ & $<0,3$ & $<0,1$ & $<0,3$ & - & $<0,1$ & - \\
\hline Ac.stéarique & C18:0 & $1-3$ & $0,5-5,0$ & $3-6$ & $4-6$ & $3-6$ & $4-6$ & $3,0-4,5$ \\
\hline Ac.arachidique & C20:0 & $<0,3$ & $<0,6$ & $<0,3$ & $<1$ & $<1$ & $<0,5$ & $0,2-0,5$ \\
\hline Ac.béhénique & C22:0 & $<0,2$ & 0,2 & $<0,5$ & $<0,3$ & $<0,7$ & $0,5-1$ & $0,5-1,1$ \\
\hline Ac.lignocérique & C24:0 & - & 0,2 & - & $<0,3$ & $<0,4$ & $<0,3$ & $<0,5$ \\
\hline \multicolumn{9}{|l|}{ mono-insaturés } \\
\hline Ac.palmitoléique & C16:1 n-7 & $<0,2$ & $0,3-3,5$ & $<0,5$ & $<0,2$ & $<0,2$ & $<0,5$ & $<0,1$ \\
\hline Ac.heptadécènoïque & C17:1 & - & $<0,3$ & $<0,1$ & - & - & - & - \\
\hline Ac.oléique & C18:1 n-9 & $14-21$ & $55-83$ & $14-22$ & $36-42$ & $17-26$ & $15-25$ & $75-85$ \\
\hline $\begin{array}{l}\text { Ac.gadoléique/ } \\
\text { gondoïque }\end{array}$ & $\begin{array}{l}\text { C20:1 n-11/ } \\
n-9\end{array}$ & $<0,3$ & 0,4 & $<0,2$ & $<0,4$ & $<0,4$ & $<0,5$ & $0,1-0,5$ \\
\hline Ac.érucique & C22:1 n-9 & - & - & - & - & $<0,2$ & $<0,2$ & $<0,1$ \\
\hline \multicolumn{9}{|l|}{ polyinsaturés } \\
\hline Ac.linoléique & C18:2 n-6 & $54-65$ & $3,5-21,0$ & $65-73$ & $39-47$ & $50-62$ & $62-70$ & $7,0-17,0$ \\
\hline Ac.alphalinolénique & C18:3 n-3 & $9-15$ & $<0,9$ & $<0,5$ & $<0,6$ & $4-10$ & $<0,2$ & $<0,3$ \\
\hline Ac.gras saturés & AGS & $7-11$ & $9-26$ & $11-17$ & 13-19 & $11-21$ & $10-16$ & $6-10$ \\
\hline Ac.gras mono-insaturés & AGMI & $14-21$ & $56-87$ & $15-23$ & $36-43$ & $17-27$ & $15-26$ & $75-83$ \\
\hline \multirow[t]{2}{*}{ Ac.gras polyinsaturés } & AGPI & $63-80$ & $4-22$ & $65-73$ & $40-48$ & $54-72$ & $62-70$ & $10-21$ \\
\hline & & & & & & & & $\begin{array}{l}\text { \& C20:2 n-6, ND-0,3 } \\
\& C 20: 4 \text { n-6, ND-0,1 }\end{array}$ \\
\hline
\end{tabular}

arrangement dense et compact des chaînes dû à des interactions spécifiques (formes $\beta$ et $\beta^{\prime}$ ) et celle associée à un arrangement plus lâche (forme $\alpha$ ) par perte de ces interactions; les formes les plus compactes étant généralement les formes les plus stables (Cansell, 2005).

Ainsi, la dureté (ou la consistance) d'un corps gras dépend i) de sa composition en acides gras; du plus dur au plus fluide : $A G S>[A G T]>A G M I>A G P I$; ii) de sa structure triglycéridique qui influence l'importance du polymorphisme à l'état solide ; iii) des conditions de sa mise en œuvre : température et passé thermique (vitesse de refroidissement, donc de cristallisation), travail mécanique (agitation, pression ou cisaillement pendant la cristallisation).

Les corps gras riches en acides gras insaturés fondent à des températures inférieures à $15{ }^{\circ} \mathrm{C}$ (huiles fluides à température ambiante-figures $2 A$ et
$B)$; ceux plus riches en AGS fondent à partir de $30{ }^{\circ} \mathrm{C}$ (graisses solides à température ambiante - figure 2C).

Dans la mesure où la matière grasse incorporée à de nombreux produits alimentaires formulés se trouve dans un état cristallisé ou semi-cristallisé aux températures de stockage et d'utilisation, elle conditionne les propriétés rhéologiques et la texture des produits finis et différemment selon qu'elle se trouve en phase continue ou dispersée : émulsions eau dans I'huile telles que le beurre et les margarines ou huile dans eau du type sauce mayonnaise, crèmes glacées ou chantilly (Cansell, 2005).

La stabilité oxydative est également dépendante de la composition en acides gras et secondairement de la structure triglycéridique. La sensibilité vis-à-vis de l'oxydation est directement reliée au nombre $d$ 'insaturations selon une progression pratiquement exponentielle (non linéaire) avec un facteur multipli- catif de l'ordre de 100 pour une chaîne linolénique (3 insaturations) comparée à une chaîne oléique mono-insaturée. Les cinétiques réactionnelles du phénomène oxydatif (chimique de type radicalaire, spontané, évolutif, irréversible et altératif) dépendent de paramètres extérieurs à la matrice grasse (température, aération, lumière) et d'autres qui lui sont propres: insaturation globale, présence i) d'antioxydants naturels (ou ajoutés), ii) de composés d'une altération préalable ou en cours (soit des acides gras libres produits par hydrolyse - plus réactifs que sous forme estérifiée dans les triglycérides -, soit des composés primaires d'oxydation - radicaux libres, hydroperoxydes) et iii) de promoteurs (traces métalliques, photosensibilisateurs).

Certains travaux ont montré qu'au sein de triglycérides modèles (TG) riches en AGPI (C18:2 et C18:3 notamment), l'oxydabilité pouvait être reliée à la proximité de chaînes réactives en posi- 
Tableau 3. Compositions en acides gras de quelques corps gras végétaux (\% des acides gras totaux) (ND : <0,05\%).

\begin{tabular}{|c|c|c|c|c|c|c|}
\hline Huiles/corps gras & & Beurre de cacao & $\begin{array}{l}\text { Beurre } \\
\text { de karité }\end{array}$ & Coprah & Palme & Palmiste \\
\hline Ac. gras & & toutes origines & & & & \\
\hline \multicolumn{7}{|l|}{ saturés } \\
\hline Ac. caproïque & C6:0 & & & ND-0,7 & - & ND-0,8 \\
\hline Ac. caprylique & C8:0 & & & $4,6-10,0$ & - & $2,4-6,2$ \\
\hline Ac. caprique & C10:0 & & & $5,0-8,0$ & - & $2,6-5,0$ \\
\hline Ac.laurique & C12:0 & & $<1$ & $45,1-53,2$ & ND-0,5 & $45,0-55,0$ \\
\hline Ac.myristique & C14:0 & $<0,3$ & $<0,7$ & $16,8-21,0$ & $0,5-2,0$ & $14,0-18,0$ \\
\hline Ac.palmitique & C16:0 & $24-30$ & $3-5$ & $7,5-10,2$ & $39,3-47,5$ & $6,5-10,0$ \\
\hline Ac.margarique & C17:0 & $<0,3$ & - & ND & $<0,2$ & ND \\
\hline Ac.stéarique & C18:0 & $30-37$ & $28-45$ & $2,0-4,0$ & $3,5-6,0$ & $1,0-3,0$ \\
\hline Ac.arachidique & C20:0 & $0,7-1,4(1)$ & $<1,5$ & ND- 0,2 & $<1,0$ & ND-0,2 \\
\hline Ac.béhénique & C22:0 & $\leq 0,2$ & - & ND & $<0,2$ & ND-0,2 \\
\hline Ac.lignocérique & C24:0 & $\leq 0,2$ & - & ND & ND & ND \\
\hline \multicolumn{7}{|l|}{ mono-insaturés } \\
\hline Ac.palmitoléique & C16:1 n-7 & $\leq 0,3$ & $<0,3$ & ND & $<0,6$ & ND-0,2 \\
\hline Ac.heptadécènoïque & C17:1 & - & - & ND & ND & ND \\
\hline Ac.oléique & C18:1 n-9 & $33-39$ & $42-59$ & $5,0-10,0$ & $36,0-44,0$ & $12,0-19,0$ \\
\hline $\begin{array}{l}\text { Ac.gadoléique/ } \\
\text { gondoïque }\end{array}$ & $\begin{array}{l}\text { C20:1 n-11/ } \\
n-9\end{array}$ & $\leq 0,2$ & - & ND-0,2 & $<0,4$ & ND-0,2 \\
\hline Ac.érucique & C22:1 n-9 & - & - & ND & ND & ND \\
\hline \multicolumn{7}{|l|}{ polyinsaturés } \\
\hline Ac.linoléique & C18:2 n-6 & $2-5$ & $3-9$ & $1,0-2,5$ & $9,0-12,0$ & $1,0-3,5$ \\
\hline Ac.alphalinolénique & C18:3 n-3 & $<0,3$ & $<1$ & ND-0,2 & $<0,5$ & ND-0,2 \\
\hline Ac.gras saturés & AGS & 61 & 35 & 91 & $45-55$ & 82 \\
\hline Ac.gras monoinsaturés & AGMI & 35 & 60 & 7 & $38-45$ & 16 \\
\hline Ac.gras polyinsaturés & AGPI & 3 & 5 & 1,8 & $9-12$ & 2 \\
\hline
\end{tabular}

Tableau 4. Teneurs en tocophérols ou tocotriénols totaux de quelques huiles végétales, formes majoritaires selon les huiles et pouvoir vitaminique $E$ global (en $\alpha$-tocophérol équivalent).

\begin{tabular}{|c|c|c|c|c|}
\hline Huile/corps gras & $\begin{array}{l}\text { Tocophérols totaux } \\
\text { (mg/100 g d'huile) }\end{array}$ & $\begin{array}{l}\text { Formes } \\
\text { majoritaires }\end{array}$ & $\begin{array}{l}\text { Pouvoir vitaminique E } \\
\text { pour } 100 \mathrm{~g} \text { d'huile } . .\end{array}$ & $\begin{array}{l}\text { Wet rang } \\
\text { correspondant }\end{array}$ \\
\hline Olive & $5-15$ & $\alpha$ & $5-10$ & 8 \\
\hline Pépins de raisin & $30-50$ & $\alpha, \gamma$ tocotriénols & $10-30$ & 7 \\
\hline Noisette & $30-55$ & $\alpha$ & $30-55$ & 3 \\
\hline Noix & $55-60$ & $\gamma$ & $5-10$ & 8 \\
\hline Colza & $60-90$ & $\gamma$ & $20-40$ & 4 \\
\hline Palme & $30-100$ & tocotriénols & $10-30$ & 7 \\
\hline Arachide & $30-100$ & $\alpha$ & $5-30$ & 5 \\
\hline Tournesol & $70-120$ & $\alpha, \gamma$ & $45-110$ & 2 \\
\hline Soja & $80-170$ & $\gamma, \delta$ & $10-40$ & 6 \\
\hline Germe de maïs & $110-185$ & $\gamma$ & $10-40$ & 6 \\
\hline Germe de blé & $300-350$ & $\alpha, \beta$ & $135-225$ & 1 \\
\hline
\end{tabular}


tions interne et externes facilitant les interactions (Miyashita, 1990).

L'intérêt nutritionnel des corps gras est directement lié aux quatre principaux rôles physiologiques des lipides: 1) source d'énergie ( $1 \mathrm{~g}$ de lipide $=9$ kcal) ; 2) rôle structural important en tant que constituants des membranes cellulaires; 3) précurseurs de molécules à haute activité biologique ou " médiateurs chimiques oxygénés" (prostaglandines, leucotriènes, thromboxanes) jouant un rôle important dans des fonctions vitales (agrégation plaquettaire et coagulation du sang, fonction rénale, phénomènes inflammatoires et immunitaires. . ) ; 4) apport et véhicule de vitamines liposolubles (E, A, D principalement). Pour le rôle structural et celui de précurseur métabolique, les acides linoléique (C18:2) et alphalinolénique (C18:3) occupent une place indispensable puisque l'organisme ne peut les synthétiser et qu'ils sont chacun précurseur de deux voies de transformations métaboliques bien distinctes, respectivement dénommées séries oméga- 6 et 3 ; ces deux voies utilisent les mêmes enzymes pour synthétiser des dérivés à plus longue chaîne, plus insaturés, à partir des deux précurseurs : il y a donc compétition entre les deux voies, avec des risques de perturbations en cas de déséquilibre des apports, ce que les recommandations nutritionnelles visent à éviter (voir plus haut).

Par ailleurs, la position occupée par les acides gras sur le glycérol joue un rôle sur la biodisponibilité des acides gras (nutriment biodisponible : fraction de la part consommée utilisable par l'organisme après digestion, acheminement aux cellules et utilisation cellulaire). Ainsi un acide gras, placé en position interne (sn-2) des triglycérides, est généralement considéré comme étant plus biodisponible parce qu'il échappe à l'action de la lipase pancréatique [spécifique des positions externes (sn-1 \& 3)] et peut ainsi être absorbé à travers la barrière intestinale sous forme de 2-monoglycérides (2-MAG). Ces derniers serviront de précurseurs pour la resynthèse dans l'entérocyte des triglycérides qui seront ensuite sécrétés (sous forme de chylomicrons) dans la lymphe puis dans le sang. Cette particularité fondée sur plusieurs études antérieures sera peut-être à nuancer suite à de récents travaux (Couëdelo et al., 2012) montrant que dans l'entérocyte, une partie des 2-MAG peut être hydrolysée au cours de l'absorption intestinale conduisant ainsi à une redistribution de l'acide gras situé en position sn-2, entre les positions interne et externes, des triglycérides synthétisés dans ces cellules intestinales.

\section{Procédés d'obtention et préservation des composés d'intérêt}

\section{Trituration}

L'extraction des huiles de graines ou de fruits oléagineux, ou trituration, regroupe les opérations mécaniques et/ou chimiques qui vont conduire au déshuilage en optimisant le rendement et la qualité de I'huile obtenue. Si le process n'emploie que des opérations mécaniques (pression), les huiles peuvent être qualifiées de vierges; elles peuvent aussi être ultérieurement raffinées partiellement (par exemple, désodorisées) ou totalement (huiles de pression raffinées).

La très grande majorité des huiles produites suit un process comportant en plus une étape d'extraction par solvant à partir du résidu de pression, appelé tourteau de pression. Pour certaines graines considérées comme peu riches en huile ( $<25 \%)$, telles que le soja ou le coton, l'étape de pré-pression mécanique n'est pas nécessaire ; I'huile produite ainsi par extraction au solvant doit systématiquement être raffinée.

La qualité de la matière première (graine ou fruit) en entrée de process est essentielle pour pouvoir garantir la qualité de l'huile produite. Les oléagineux doivent donc être stockés le moins longtemps possible (surtout pour les fruits à cause de leur teneur en eau qui limite la conservation) dans de bonnes conditions (température, humidité faible, aération) pour éviter hydrolyse et oxydation (Pagès, 2009).

Le mode d'obtention, par pression avec ou sans conditionnement thermique des graines influence l'aspect (couleur) et les teneurs en phospholipides et en constituants mineurs : la pression à froid favorise l'extraction des triglycérides en limitant celle des phospholipides $(\mathrm{PL})$ contrairement à une pression avec conditionnement thermique qui aug- ment les quantités extraites de PL (Carré, 2010).

La comparaison d'huiles obtenues par pression et par extraction au solvant révèle que ce dernier procédé produit des huiles plus riches en composés mineurs (stérols, tocophérols) et en phospholipides (Carré, 2010).

\section{Raffinage}

Le raffinage a pour but de maintenir ou d'améliorer les caractères organoleptiques (goût et odeur neutres, limpidité, couleur jaune clair), nutritionnels et la stabilité des corps gras. Pour ce faire, il met en œuvre plusieurs étapes pour éliminer des composés indésirables (gommes, cires, acides gras libres, pigments, traces métalliques, composés odorants volatils) et les contaminants potentiellement présents dans les matières premières, tout en maîtrisant la formation de nouveaux composés indésirables par hydrolyse, oxydation ou isomérisation. La conduite du procédé peut au besoin s'adapter aux usages ultérieurs qui seront faits des huiles raffinées produites: ainsi, un usage alimentaire (ou cosmétique/ pharmaceutique) conduira le raffineur à optimiser son procédé afin de conserver les constituants d'intérêt nutritionnel (acides gras polyinsaturés, vitamine $\mathrm{E}$ ).

Il existe deux types de raffinage: chimique et " physique » qu'il conviendrait mieux d'appeler "distillation neutralisante ". Le raffinage chimique élimine les acides gras libres par une étape de neutralisation à la soude; la distillation neutralisante (DN) élimine ces composés indésirables (désacidification) par distillation sous vide poussé avec injection de vapeur (Pagès, 2010).

Le maintien des composés d'intérêt nutritionnel (AGPI et vitamine E principalement) au cours de ces process sera assuré en maîtrisant particulièrement les étapes de décoloration (risque d'isomérisation des AGPI au contact des terres) et de désodorisation (ou DN) selon le type de raffinage pratiqué (risques d'isomérisation géométrique des AGPI, risques de pertes des phytostérols et vitamine $\mathrm{E})$. Cette nécessaire maîtrise du procédé doit intégrer les aspects de sécurité sanitaire et garantir que le produit raffiné est exempt de toute trace des contaminants potentiels des corps gras et conforme aux limites fixées par la réglementation; sur ce plan, les 
étapes de décoloration et de désodorisation (ou DN) sont souvent importantes sinon décisives (Pagès, 2010). Enfin, à chaque étape, les risques d'altération, soit par hydrolyse (ou saponification lors de la neutralisation d'un raffinage chimique), soit par oxydation, doivent être maîtrisés ; l'injection d'un gaz inerte (azote) est parfois pratiqué en sortie de désodorisation, aux fins d'élimination de l'oxygène dissous dans l'huile pour retarder le phénomène naturel d'auto-oxydation.

\section{Procédés \\ de transformation et élargissement des fonctionnalités}

Les procédés de transformation des corps gras ont deux objectifs principaux : 1) répondre à un besoin fonctionnel de matières grasses concrètes lié aux propriétés évoquées plus haut (point 2) en matière de texturation par cristallisation et plus généralement pour assurer des propriétés de consistance, de plasticité, des caractères croustillant, croquant, etc. ; 2) améliorer la stabilité vis-à-vis des altérations oxydative et thermo-oxydative au cours des différentes utilisations.

Il existe trois procédés de transformation autorisés en alimentaire : I'hydrogénation, le fractionnement et I'interestérification.

L'hydrogénation est une transformation chimique qui a pour objectif de durcir une huile végétale et pour principe de fixer de I'hydrogène sur les doubles liaisons des acides gras insaturés en présence d'un catalyseur, généralement du nickel. Selon le point auquel est conduite la réaction, on obtient des produits partiellement hydrogénés à différents taux, caractérisés par des pourcentages augmentés de teneur en solide à une température donnée et des indices d'iode réduits; si la réaction est menée à son terme, tous les acides gras insaturés ont été transformés en AGS (hydrogénation totale). Selon les conditions mises en œuvre (niveau de frâ̂cheur du catalyseur, température de la réaction), l'hydrogénation partielle des doubles liaisons s'accompagne de la formation plus ou moins importante d'isomères géométriques trans (AGT, principalement monoinsaturés). Par sa nature même, un corps gras totalement hydrogéné ne contient plus d'AGT.

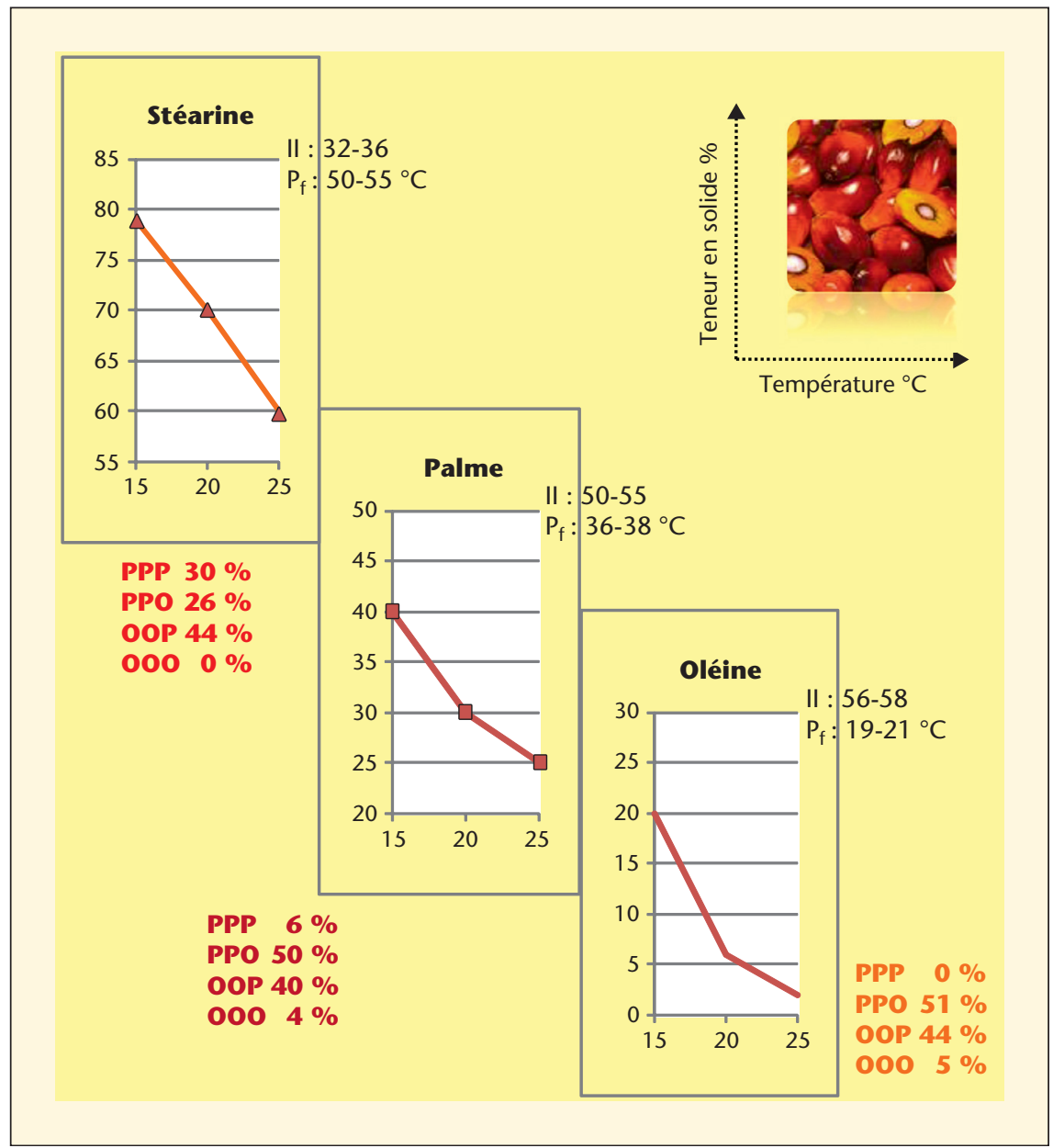

Figure 3. Fractionnement de I'huile de palme-Caractéristiques comparées de teneurs en solide en fonction de la température pour I'huile de départ et les fractions fluide (oléine/80 \%) et solide (stéarine/ $20 \%$ ) obtenues par cristallisation à $28^{\circ} \mathrm{C}$. Légende : $P=$ acide palmitique et $O=$ acide oléique.

Étant donné les risques au niveau cardiovasculaire liés à une consommation excessive d'AGT, des solutions technologiques alternatives ont commencé à se mettre en place dès la fin des années 1990 pour minimiser, voire réduire à zéro, la teneur en acides gras trans des produits; les recommandations nutritionnelles puis, dans certains pays (Danemark, États-Unis, Canada. . .), des dispositions réglementaires ont renforcé cette tendance; aujourd'hui en France, les niveaux de consommations d'AGT demeurent inférieurs au seuil recommandé par l'ANSES ${ }^{2}(<2 \%$ de I'apport énergétique total) en 2005, confirmé en 2009. À I'heure actuelle, la

\footnotetext{
${ }^{2}$ Agence nationale de sécurité sanitaire de I'alimentation, de l'environnement et du travail.
}

technologie de I'hydrogénation partielle est en nette perte de vitesse dans le secteur alimentaire.

Le fractionnement est un procédé physique qui consiste à faire cristalliser par un refroidissement selon un barème établi, les triglycérides les plus riches

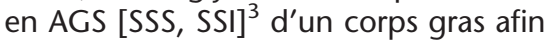
de pouvoir séparer une fraction solide ou concrète ("stéarine ") de la fraction fluide ou liquide (" oléine") n'ayant pas cristallisé dans les conditions adoptées étant donné sa composition du type [SII, $\mathrm{III}]^{4}$. L'huile de palme $\left(P_{\mathrm{F}}: 33-39{ }^{\circ} \mathrm{C}\right)$ par

\footnotetext{
${ }^{3}$ SSS : triglycéride contenant 3 AGS; SSI : triglycéride contenant 2 AGS et 1 acide gras insaturé.

${ }^{4}$ SII : triglycéride contenant 1 AGS et 2 acides gras insaturés; III : triglycéride contenant 3 acides gras insaturés.
} 
sa composition est particulièrement adaptée au fractionnement qui produit une oléine $\left(P_{F}: 22-24{ }^{\circ} \mathrm{C}\right)$ et une stéarine $\left(P_{F}: 40-50{ }^{\circ} \mathrm{C}\right)$; il est même possible de fractionner ces premières fractions pour disposer ainsi d'une gamme de fractions dont les points de fusion s'échelonnent de moins de $20{ }^{\circ} \mathrm{C}$ ("super oléine") à plus de $50{ }^{\circ} \mathrm{C}$ (" stéarine dure "), toutefois avec des rendements beaucoup plus faibles pour ces sous-fractions.

La figure 3 présente les caractéristiques de teneurs en solide en fonction de la température, les $P_{F}$ et indices d'iode (proportionnel à l'insaturation) pour I'huile de palme et ses deux premières fractions.

L'interestérification est une transformation qui a pour objet de modifier la structure des triglycérides - donc de modifier les propriétés rhéologique du corps gras de départ - en redistribuant les acides gras sur le glycérol; le procédé peut être conduit par voie chimique (dans ce cas, la redistribution se fait au hasard ou partiellement dirigée) ou enzymatique (la spécificité des lipases permettant de ne redistribuer que les positions externes des triglycérides en préservant l'acide gras en position sn-2). Cette modification de structure permet d'obtenir un produit interestérifié de même composition en acide gras que le corps gras de départ (sauf si l'interestérification est menée entre deux huiles différentes) mais avec un ratio solide/liquide modifié.

II est donc techniquement possible de répondre à la nécessité d'une "fonction solide " et à un objectif de stabilité par un choix approprié de matières premières (huiles fluides ou semi-fluides, graisses concrètes), la combinaison de plusieurs procédés ne générant pas $\mathrm{d}^{\prime}$ acides gras trans (hydrogénation totale, fractionnement, interestérification) (Kellens, 1998 ; Van Duijn, 2000 et 2005 ; Morin, 2007).

Les solutions se réduisent lorsque des matières premières sont mises en cause et qu'elles répondent de façon adaptée aux critères fonctionnels requis pour des applications particulières; c'est aujourd'hui le cas de I'huile de palme. Les alternatives ne sont pas toujours simples à trouver, en particulier quand des caractéristiques précises de cristallisa- tion sont nécessaires à la qualité du produit fini. De même, quand il $s$ 'agit de concilier les recommandations en matière de nutrition santé (réduction des teneurs en matière grasse et réduction des acides gras saturés plutôt au bénéfice de lipides insaturés) sans que soit modifiée la qualité organoleptique des produits finis ce qui a minima peut requérir une adaptation des lignes de fabrication...

Retenons pour finir que les corps gras ont de multiples atouts au service des secteurs alimentaires qui les emploient : ils remplissent leur fonction nutritionnelle par leurs quatre importants rôles biologiques en apportant énergie et nutriments (que leur diversité permet d'équilibrer) ; ils apportent consistance et texture grâce à leurs propriétés rhéologiques, ainsi que leurs goûts et odeurs (flaveur propre ou support $d$ 'arômes) et constituent par ailleurs un excellent medium de cuisson et de friture.

L'éclairage apporté sur les principaux vecteurs de lipides dans l'alimentation (voir encadré 1 par Cécile Rauzy) permet également $d$ 'identifier les secteurs aux impacts les plus forts et de constater qu'en matière d'équilibrage nutritionnel global, les huiles végétales et les margarines ont un rôle important à jouer : contribuant pour près de $15 \%$ aux apports lipidiques, elles participent aux consommations d'AGMI, d'AGPI et d'AGS pour respectivement près de $22 \%, 28 \%$ et $6,1 \%$.

Remerciements. Les auteurs remercient Carole Vaysse et Leslie Couëdelo pour leur aide précieuse à l'exposé des récents développements des travaux de I'Unité Nutrition Métabolisme et Santé de I'ITERG.

\section{RÉFÉRENCES}

ANC. Avis de I'Agence française de sécurité sanitaire des aliments relatif à l'actualisation des apports nutritionnels conseillés pour les acides gras. AFSSA - Saisine $\mathrm{n}^{\circ}$ 2006-SA-0359 - Avis 1er mars 2010.

Cancell M. Impact de la cristallisation des corps gras sur les propriétés de produits finis. Oléagineux Corps gras Lipides 2005 ; $12: 427-31$.
Carré P. Communication personnelle - Programme Optim'Oils. Non publié, 2010.

Couëdelo L, Vaysse C, Vaique E, et al. The fraction of $\alpha$-linolenic acid present in the sn-2 position of structured triacylglycerols decreases in lymph chylomicrons and plasma triacylglycerols during the course of lipid absorption in rats. J Nutr 2012; 142 : 70-5.

Dubois V, Breton S, Linder M, et al. Proposition de classement des sources végétales d'acides gras en fonction de leur profil nutritionnel. Oléagineux Corps gras Lipides $2008 ; 15: 56-75$.

Joffre $F$, Brenne E, Lacoste F. Effect of the geographical production area of extra virgin olive oil on the polyphenol and CoQ10 contents. Communication par poster; $9^{\text {th }}$ Euro Fed Lipid Congress, 18-21/9/2011, Rotterdam.

Kellens M. Etat des lieux et évaluation des procédés de modification des matières grasses par combinaison de l'hydrogénation, de l'interestérification et du fractionnement $1^{\text {ère }}$ partie. Oléagineux Corps gras Lipides $1998 ; 5$ : 384-91.

Landrier JF. Vitamine $\mathrm{E}$ et physiologie du tissu adipeux. Oléagineux Corps gras Lipides 2011 ; $18: 83-7$.

Littarru GP, Lambrechts P. Coenzyme Q10 : multiple benefits in one ingredient. Oléagineux Corps gras Lipides 2011 ; 18 : 76-82.

Miyashita K, Frankel EN, Neff WE. Autoxidation of polyunsaturated triacylglycerols. III. Synthetic triacylglycerols containing linoleate and linolenate. Lipids $1990 ; 25$ : 4853.

Morin $\mathrm{O}$. Huiles végétales et margarines : évolution de la qualité - Les solutions technologiques à la réduction des acides gras trans. Cah Nutr Diet 2007 ; 42 : 247-53.

Pagès $X$, Morin $O$. Industries des corps gras. In : De Reynal B, Multon JL (Eds), Additifs et auxiliaires de fabrication dans les industries agroalimentaires. Paris, Lavoisier, 2009.

Pagès $X$, Morin $O$, Birot $C$ et al. Raffinage des huiles et des corps gras et élimination des contaminants. Oléagineux Corps gras Lipides 2010 ; 17 : 86-99.

Rodriguez-Acuňa R, Brenne E, Lacoste $F$. Determination of Coenzyme Q10 and Q9 in vegetable oils. J Agric Food Chem 2008 ; 56 : 6241-5.

Van Duijn G. Technical aspects of trans reduction in margarines. Oléagineux Corps gras Lipides $2000 ; 7$ : 95-98.

Van Duijn G. Technical aspects of trans reduction in modified fats. Oléagineux Corps gras Lipides 2005 ; 12 : 422-6. 


\section{Annexe 1}

\section{Les principaux aliments vecteurs de lipides dans I'alimentation}

\section{Cécile Rauzy}

Directeur Qualité-Nutrition de I'ANIA (Association nationale des industries alimentaires), 21 rue Leblanc 75015 Paris

crauzy@ania.net

L'étude INCA $2^{1}$, réalisée en 2006-2007, montre que les apports lipidiques sont en moyenne de 75 g/j chez les enfants de 3 à 17 ans et de $89 \mathrm{~g} / \mathbf{j}$ chez les adultes de 18 à 79 ans. Leur contribution à l'apport énergétique quotidien varie entre $37 \%$ et $40 \%$ selon les classes d'âge et le sexe, sachant que I'ANSES recommande dans ses nouveaux apports nutritionnels conseillés (ANC) publiés en mars 2010, un apport en lipides totaux de 35 à $40 \%$ de l'apport énergétique chez l'adulte pour un apport énergétique proche de $2000 \mathrm{kcal}^{2}$. Par contre, il faut souligner que parmi ces lipides totaux, la répartition observée dans l'étude INCA 2 entre les différents acides gras reste assez éloignée de celle préconisée dans les ANC. En effet, les apports en acides gras saturés (AGS) sont nettement plus élevés que les ANC (45\% contre $25 \%$ recommandés) alors que ceux en acides gras mono-insaturés (AGMI) sont inférieurs aux ANC (39\% au lieu des $60 \%$ recommandés).

D'après les données INCA 2(figure 4), les principaux groupes d'aliments vecteurs des lipides totaux sont, chez les adultes ${ }^{3}$, les huiles $(11,9 \%)$, les fromages $(9,8 \%)$, les beurres $(9,4 \%)$, les charcuteries (7,7 \%) et les pâtisseries et gâteaux (6,7 \%). Ces 5 groupes sont sources de 45,5\% des lipides totaux. Viennent ensuite les viandes, les plats composés et les condiments et sauces. L'ensemble des graisses "visibles ${ }^{4}$ (beurres, huiles, margarines, sauces et condiments) apporte $28 \%$ des lipides totaux ; l'ensemble « viandes, volailles, charcuteries et abats » en apporte $17 \%$, les produits laitiers (laits, fromages et ultra-frais laitiers) environ $14 \%$ et les « pâtisseries, gâteaux, biscuits, viennoiseries » $11 \%$.

Figure 4. Contribution des aliments aux lipides totaux, selon INCA 2 - Adultes

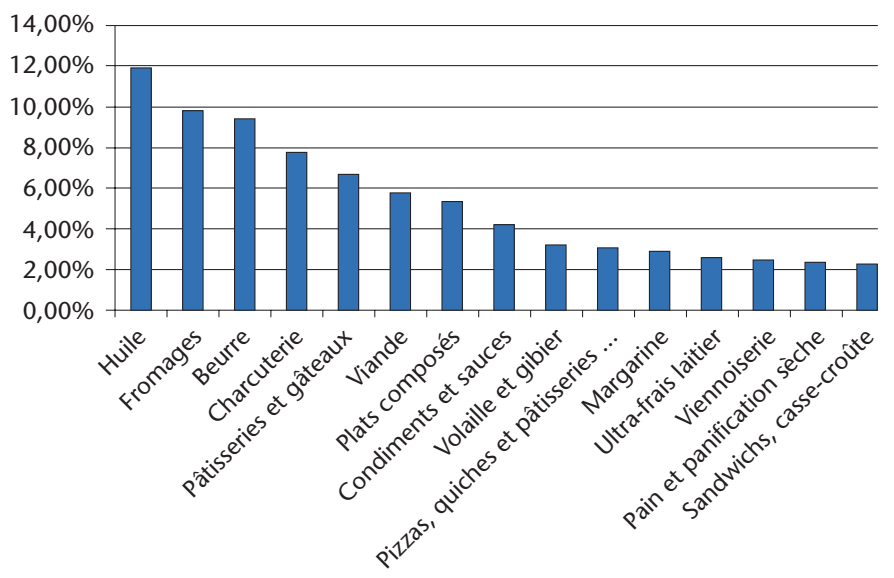

Chez les enfants (figure 5), la contribution des principaux groupes d'aliments contributeurs aux apports en lipides est globalement similaire, mais dans des proportions différentes: huiles (9,2\%), pâtisseries et gâteaux $(8,3 \%)$, beurres $(8,1 \%)$, charcuteries $(7 \%)$ et fromages $(6,7 \%)$. Ces 5 groupes sont sources de près de $40 \%$ des lipides totaux. Viennent ensuite les viandes, les plats composés et les chocolats et dérivés. L'ensemble des graisses «visibles " apporte $22 \%$ des lipides totaux ; l'ensemble "pâtisseries, gâteaux, biscuits, viennoiseries » en apporte $17 \%$, les «viandes, volailles, charcuteries et abats» $15 \%$ et les produits laitiers environ $14 \%$.

\footnotetext{
1 Étude individuelle nationale des Consommations Alimentaires 2 (INCA 2), 2006-2007), AFSSA, février 2009.

${ }^{2}$ Avis de l'AFSSA du 1 er mars 2010 relatif à l'actualisation des apports nutritionnels conseillés pour les acides gras

${ }^{3}$ Rapport du groupe PNNS sur les lipides - Septembre 2009

${ }^{4} \mathrm{Ne}$ tient compte que des consommations de corps gras en tartine, et en ajout directement par le sujet (cuisson, service et assaisonnement des viandes, des poissons, des féculents, des légumes, des œufs). N'inclut pas les matières grasses utilisées comme ingrédient d'une recette (beurre des gâteaux...) ou comme composant d'un aliment complexe (huile des frites).
} 
Figure 5. Contribution des aliments aux lipides totaux, selon INCA 2 - Enfants 3-17 ans

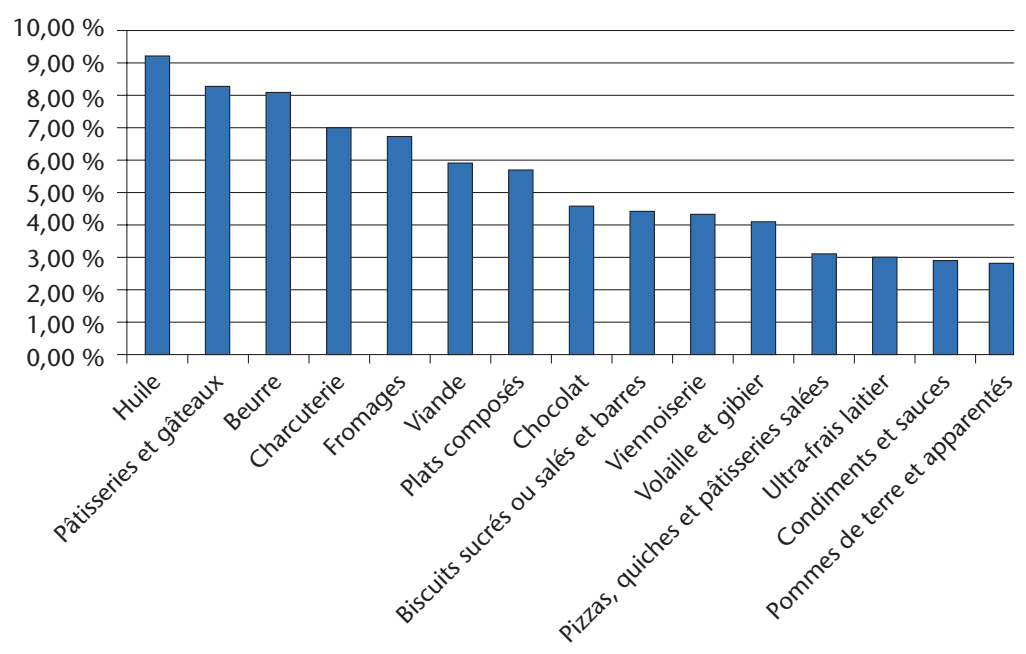

Comme le montre le tableau 5, réalisé par l'ANSES dans le cadre du groupe de travail PNNS sur les lipides, chez les adultes les 5 principaux groupes d'aliments contributeurs aux apports en AGS, AGMI et AGPI sont globalement similaires, mais interviennent dans des proportions différentes :

- les AGMI sont apportés majoritairement par les huiles $(19,2 \%)$, les charcuteries $(9,6 \%)$, les viandes $(7,1 \%)$, les fromages $(7,1 \%)$ et les beurres (6,9\%). Ces 5 groupes sont donc sources de $50 \%$ des apports en AGMI ;

- les AGPI sont apportés principalement par les huiles (21,5\%), les condiments et sauces $(12,3 \%)$, les margarines $(6,6 \%)$, les charcuteries $(5,8 \%)$, les plats composés $(5,8 \%)$. Ces 5 groupes apportent plus de la moitié des AGPI (52\%);

- les AGS sont apportés par les beurres (15,6\%), les fromages (15,5\%), les pâtisseries et gâteaux $(9,0 \%)$, les charcuteries $(7,3 \%)$ et les viandes $(5,6 \%)$. Ces 5 groupes sont donc sources de $53 \%$ des apports en acides gras saturés.

\section{Tableau 5}

Apports en lipides et en acides gras mono-insaturés, polyinsaturés et saturés des 43 groupes d'aliments et leurs contributions chez les adultes de 18-79 ans $(n=1918)$ (pour chaque nutriment, en gras : les 5 groupes d'aliments principaux contributeurs en ce nutriment).

\begin{tabular}{|l|l|l|l|l|l|l|l|l|}
\hline & \multicolumn{2}{|c|}{ Lipide } & \multicolumn{2}{|c|}{ AGMI } & \multicolumn{3}{|c|}{ AGPI } & \multicolumn{1}{|c|}{ AGS } \\
\hline & $\mathbf{g} / \mathbf{j}$ & $\%$ & $\mathbf{g} / \mathbf{j}$ & $\mathbf{\%}$ & $\mathbf{g} / \mathbf{j}$ & $\%$ & $\mathbf{g} / \mathbf{j}$ & \multicolumn{1}{|c|}{$\%$} \\
\hline Huile & $\mathbf{1 0 , 7}$ & $\mathbf{1 1 , 9} \%$ & $\mathbf{6 , 1}$ & $\mathbf{1 9 , 2} \%$ & $\mathbf{2 , 9}$ & $\mathbf{2 1 , 5} \%$ & 1,4 & $3,9 \%$ \\
\hline Fromages & $\mathbf{8 , 8}$ & $\mathbf{9 , 8} \%$ & $\mathbf{2 , 3}$ & $\mathbf{7 , 1} \%$ & 0,3 & $2,1 \%$ & $\mathbf{5 , 6}$ & $\mathbf{1 5 , 5} \%$ \\
\hline Beurre & $\mathbf{8 , 4}$ & $\mathbf{9 , 4} \%$ & $\mathbf{2 , 2}$ & $\mathbf{6 , 9} \%$ & 0,3 & $2,4 \%$ & $\mathbf{5 , 7}$ & $\mathbf{1 5 , 6} \%$ \\
\hline Charcuterie & $\mathbf{6 , 9}$ & $\mathbf{7 , 7} \%$ & $\mathbf{3 , 1}$ & $\mathbf{9 , 6} \%$ & $\mathbf{0 , 8}$ & $\mathbf{5 , 8} \%$ & $\mathbf{2 , 7}$ & $\mathbf{7 , 3} \%$ \\
\hline Pâtisseries et gâteaux & $\mathbf{6 , 0}$ & $\mathbf{6 , 7} \%$ & 1,7 & $5,4 \%$ & 0,5 & $4,0 \%$ & $\mathbf{3 , 3}$ & $\mathbf{9 , 0} \%$ \\
\hline Viande & 5,1 & $5,8 \%$ & $\mathbf{2 , 3}$ & $\mathbf{7 , 1} \%$ & 0,4 & $2,9 \%$ & $\mathbf{2 , 0}$ & $\mathbf{5 , 6} \%$ \\
\hline Plats composés & 4,8 & $5,3 \%$ & 1,9 & $5,8 \%$ & $\mathbf{0 , 8}$ & $\mathbf{5 , 8} \%$ & 1,6 & $4,4 \%$ \\
\hline Condiments et sauces & 3,7 & $4,2 \%$ & 1,3 & $4,0 \%$ & $\mathbf{1 , 6}$ & $\mathbf{1 2 , 3} \%$ & 0,7 & $1,9 \%$ \\
\hline Volaille et gibier & 2,8 & $3,2 \%$ & 1,1 & $3,5 \%$ & 0,6 & $4,5 \%$ & 0,8 & $2,2 \%$ \\
\hline Pizzas, quiches et pâtisseries salées & 2,7 & $3,0 \%$ & 1,1 & $3,4 \%$ & 0,3 & $2,0 \%$ & 1,1 & $2,9 \%$ \\
\hline Margarine & 2,6 & $2,9 \%$ & 0,8 & $2,6 \%$ & $\mathbf{0 , 9}$ & $\mathbf{6 , 6} \%$ & 0,8 & $2,2 \%$ \\
\hline Ultra-frais laitier & 2,3 & $2,6 \%$ & 0,6 & $2,0 \%$ & 0,1 & $0,5 \%$ & 1,4 & $3,9 \%$ \\
\hline Viennoiserie & 2,2 & $2,5 \%$ & 0,6 & $1,9 \%$ & 0,2 & $1,4 \%$ & 1,1 & $3,1 \%$ \\
\hline Pain et panification sèche & 2,2 & $2,4 \%$ & 0,4 & $1,1 \%$ & 0,6 & $4,3 \%$ & 0,4 & $1,1 \%$ \\
\hline Sandwichs, casse-croûte & 2,1 & $2,3 \%$ & 0,7 & $2,3 \%$ & 0,3 & $2,0 \%$ & 1,0 & $2,6 \%$ \\
\hline Fufs et dérivés & 1,9 & $2,2 \%$ & 0,8 & $2,4 \%$ & 0,3 & $2,4 \%$ & 0,6 & $1,6 \%$ \\
\hline Pommes de terre et apparentés & 1,9 & $2,1 \%$ & 0,5 & $1,5 \%$ & 0,2 & $1,4 \%$ & 0,6 & $1,7 \%$ \\
\hline
\end{tabular}




\begin{tabular}{|c|c|c|c|c|c|c|c|c|}
\hline Biscuits sucrés ou salés et barres & 1,8 & $2,0 \%$ & 0,6 & $2,0 \%$ & 0,2 & $1,5 \%$ & 0,9 & $2,4 \%$ \\
\hline Poissons & 1,8 & $2,0 \%$ & 0,6 & $1,9 \%$ & 0,6 & $4,4 \%$ & 0,4 & $1,0 \%$ \\
\hline Chocolat & 1,7 & $1,9 \%$ & 0,7 & $2,2 \%$ & 0,1 & $1,0 \%$ & 0,8 & $2,2 \%$ \\
\hline Lait & 1,4 & $1,6 \%$ & 0,4 & $1,3 \%$ & 0,0 & $0,3 \%$ & 0,9 & $2,3 \%$ \\
\hline Entremets, crèmes desserts et laits gélifiés & 1,4 & $1,6 \%$ & 0,3 & $1,1 \%$ & 0,1 & $0,6 \%$ & 0,8 & $2,1 \%$ \\
\hline Légumes (hors pommes de terre) & 1,3 & $1,4 \%$ & 0,5 & $1,6 \%$ & 0,3 & $2,6 \%$ & 0,2 & $0,6 \%$ \\
\hline Glaces et desserts glacés & 0,9 & $1,0 \%$ & 0,2 & $0,6 \%$ & 0,0 & $0,3 \%$ & 0,6 & $1,7 \%$ \\
\hline Fruits secs et graines oléagineuses & 0,8 & $0,9 \%$ & 0,3 & $1,0 \%$ & 0,3 & $2,4 \%$ & 0,1 & $0,3 \%$ \\
\hline Soupes et bouillons & 0,7 & $0,8 \%$ & 0,2 & $0,7 \%$ & 0,2 & $1,2 \%$ & 0,2 & $0,6 \%$ \\
\hline Céréales pour petit déjeuner & 0,3 & $0,4 \%$ & 0,1 & $0,3 \%$ & 0,1 & $0,4 \%$ & 0,2 & $0,5 \%$ \\
\hline Fruits & 0,4 & $0,4 \%$ & 0,0 & $0,1 \%$ & 0,1 & $0,8 \%$ & 0,0 & $0,1 \%$ \\
\hline Café & 0,2 & $0,3 \%$ & 0,1 & $0,3 \%$ & 0,0 & $0,1 \%$ & 0,1 & $0,3 \%$ \\
\hline Pâtes & 0,3 & $0,3 \%$ & 0,1 & $0,2 \%$ & 0,1 & $0,8 \%$ & 0,1 & $0,2 \%$ \\
\hline Autres boissons chaudes & 0,2 & $0,2 \%$ & 0,1 & $0,2 \%$ & 0,0 & $0,0 \%$ & 0,1 & $0,3 \%$ \\
\hline Abats & 0,2 & $0,2 \%$ & 0,1 & $0,2 \%$ & 0,0 & $0,2 \%$ & 0,1 & $0,2 \%$ \\
\hline Boissons fraîches sans alcool & 0,2 & $0,2 \%$ & 0,0 & $0,1 \%$ & 0,0 & $0,3 \%$ & 0,0 & $0,1 \%$ \\
\hline Riz et blé dur ou concassé & 0,1 & $0,1 \%$ & 0,0 & $0,1 \%$ & 0,0 & $0,2 \%$ & 0,0 & $0,1 \%$ \\
\hline Crustacés et mollusques & 0,1 & $0,1 \%$ & 0,0 & $0,1 \%$ & 0,0 & $0,2 \%$ & 0,0 & $0,1 \%$ \\
\hline Sucres et dérivés & 0,1 & $0,1 \%$ & 0,0 & $0,1 \%$ & 0,0 & $0,2 \%$ & 0,0 & $0,1 \%$ \\
\hline Autres graisses & 0,1 & $0,1 \%$ & 0,1 & $0,2 \%$ & 0,0 & $0,1 \%$ & 0,1 & $0,1 \%$ \\
\hline Légumes secs & 0,1 & $0,1 \%$ & 0,0 & $0,0 \%$ & 0,0 & $0,2 \%$ & 0,0 & $0,0 \%$ \\
\hline ADAP & 0,1 & $0,1 \%$ & 0,0 & $0,0 \%$ & 0,0 & $0,2 \%$ & 0,0 & $0,0 \%$ \\
\hline Autres céréales & 0,0 & $0,0 \%$ & 0,0 & $0,0 \%$ & 0,0 & $0,1 \%$ & 0,0 & $0,0 \%$ \\
\hline Compotes et fruits cuits & 0,0 & $0,0 \%$ & 0,0 & $0,0 \%$ & 0,0 & $0,1 \%$ & 0,0 & $0,0 \%$ \\
\hline Eaux & 0,0 & $0,0 \%$ & 0,0 & $0,0 \%$ & 0,0 & $0,0 \%$ & 0,0 & $0,0 \%$ \\
\hline Boissons alcoolisées & 0,0 & $0,0 \%$ & 0,0 & $0,0 \%$ & 0,0 & $0,0 \%$ & 0,0 & $0,0 \%$ \\
\hline TOTAL & 89,3 & $100,0 \%$ & 32,0 & $100,0 \%$ & 13,4 & $100,0 \%$ & 36,4 & $100,0 \%$ \\
\hline
\end{tabular}

Source : Rapport du groupe PNNS sur les lipides et étude INCA 22006 -2007, A FSSA 